\title{
Progressive recovery of a tropical deforested stream community after a flash flood
}

\author{
Recuperação gradual de uma comunidade de riacho \\ tropical desflorestado após enchente repentina
}

\section{Lucas Cerqueira Marques, Mônica Ceneviva-Bastos and Lilian Casatti}

Departamento de Zoologia e Botânica, Universidade Estadual Paulista - UNESP, Rua Cristóvão Colombo, 2265, CEP 15054-000, São José do Rio Preto, SP, Brazil e-mail: marques_bio@yahoo.com.br; mcbastos@gmail.com; licasatti@gmail.com

\begin{abstract}
Aim: In this study, we evaluated and compared community attributes from a tropical deforested stream, located in a pasture area, in a period before (PRED I) and three times after (POSD I, II, and III) a flash flood, in order to investigate the existence of temporal modifications in community structure that suggests return to conditions previous to the flash flood. Methods: Biota samples included algae, macrophytes, macroinvertebrates, and fish assemblages. Changes in stream physical structure we also evaluated. Similarity of the aquatic biota between pre and post-disturbance periods was examined by exploratory ordination, known as Non-Metric Multidimensional Scaling associated with Cluster Analysis, using quantitative and presence/absence Bray-Curtis similarity coefficients. Presence and absence data were used for multivariate correlation analysis (Relate Analysis) in order to investigate taxonomic composition similarity of biota between pre and post-disturbance periods. Results: Our results evidenced channel simplification and an expressive decrease in richness and abundance of all taxa right after the flood, followed by subsequent increases of these parameters in the next three samples, indicating trends towards stream community recovery. Bray-Curtis similarity coefficients evidenced a greater community structure disparity among the period right after the flood and the subsequent ones. Multivariate correlation analysis evidenced a greater correlation between macroinvertebrates and algae/macrophytes, demonstrating the narrow relation between their recolonization dynamics. Conclusions: Despite overall community structure tended to return to previous conditions, recolonization after the flood was much slower than that reported in literature. Finally, the remarkably high flood impact along with the slow recolonization could be a result of the historical presence of anthropic impacts in the region, such as siltation, riparian forest complete depletion, and habitat simplification, which magnified the effects of a natural disturbance.
\end{abstract}

Keywords: disturbance, erosive floods, recolonization, macroinvertebrates, macrophytes, fish.

Resumo: Objetivo: Neste estudo, foram avaliados e comparados os atributos de uma comunidade de riacho no Brasil em um período anterior (PRED) e três posteriores (POSD I, II e III) a uma enchente repentina, a fim de investigar a existência de modificaçôes temporais na estrutura da comunidade que sugira retorno às condiçóes anteriores à enchente. Métodos: Amostras da biota incluíram algas, macrófitas, macroinvertebrados e peixes. Alteraçóes na estrutura física do riacho também foram avaliadas. A similaridade da biota aquática entre os períodos pré e pós-distúrbio foi examinada por ordenação exploratória, conhecida como Análise de Escalonamento Multidimensional Não Métrico com Cluster, utilizando os coeficientes de similaridade de Bray-Curtis quantitativo e de presença/ausência. Dados de presença e ausência foram usados para análise de correlaçáo multivariada (Relate Analysis) a fim de investigar a similaridade da composição taxonômica entre os períodos pré e pós-distúrbio. Resultados: Houve uma diminuição do canal do riacho e expressivo decréscimo na riqueza e abundância de todos os táxons logo após a enchente, seguido por aumentos subsequentes nas três próximas amostragens, indicando uma tendência em direção à recuperação da comunidade de riacho. Os coeficientes de Bray-Curtis evidenciaram grande disparidade na estrutura da comunidade entre o período imediatamente após o distúrbio e os subsequentes. A análise de correlação multivariada demonstrou forte correlação entre macroinvertebrados e algas/macrófitas, indicando estreita relação entre a dinâmica de recolonização desses grupos. Conclusóes: Apesar da estrutura da comunidade indicar retorno às condiçôes iniciais, a recolonização foi 
muito mais lenta em relaçáo aos registros da literatura. Finalmente, o forte impacto da enchente, somado à recolonização lenta, podem ser o resultado da presença histórica de interferências antrópicas na região, como assoreamento, destruiçâo completa da vegetação ripária e simplificação do hábitat, que amplificaram os efeitos de um distúrbio natural.

Palavras-chave: distúrbio, enchentes erosivas, recolonização, macroinvertebrados, macrófitas, peixes.

\section{Introduction}

Composition and species richness within a community can be seen as a result from the interaction of biotic and abiotic processes, and the comprehension of these processes has a great ecological importance (Thomson et al., 2002). However, disturbances can affect community organization, leading to changes in ecological and evolutionary processes (Stanley et al., 2010). Among the agents of natural disturbances in streams, floods are the most evident and frequent (Thomson et al., 2002; Gibbins et al., 2007) and can be an extremely important event for the maintenance of ecological integrity and biological productivity by becoming primary sources of environmental variability (Poff et al., 1997; Jurajda et al., 2006).

Erosive floods (sensu Matthews, 1998), however, can cause a large destructive impact on stream physical habitat (Lytle, 2000), modifying channel morphology and substrate types (Yount and Niemi, 1990). Among erosive floods, flash floods are those marked by a very quick discharge rise, overflowing into the channel and also receding rapidly (Henry et al., 1994). Generally, these changes reflect in decreases in richness and abundance of producers (Henry et al., 1994; Barrat-Segretain and Amoros, 1995; Cellot et al., 1998) and consumers (Thomson et al., 2002; Franssen et al., 2006; Jurajda et al., 2006; Pires et al., 2008), resulting in modifications in the whole trophic structure (Death, 2010).

In this context, stream recovery process implies in the return of the system to conditions present before the disturbance, since disturbance itself is no longer acting (Yount and Niemi, 1990). In this context, every environmental stress aspects, like the ecosystem response to the disturbance, as well as the way in which they adapt and recover, are of fundamental importance (Longing and Haggard, 2010). Additionally, the recolonization speed of all biota can also depend on the auto-ecological characteristics of each remaining species, such as life history attributes (Yount and Niemi, 1990), availability and accessibility to refugia, resistance (ability to withstand the disturbance, maintaining the same conditions during and after the event), and resilience (ability to recover after the disturbance)
(Pickett and White, 1985; Grimm and Fisher, 1989; Lytle, 2000; Lake, 2013), in response to natural and anthropic impacts. This recovery ability has been considered an important attribute for stream integrity, as approaches involving these two components (resistance/resilience) are traditional in stream ecology (Lake, 2013).

In view of the wide universe of impacts that streams in northwestern Sao Paulo State have been exposed (Casatti et al., 2009), along with the difficulty and the need of distinguishing patterns derived from anthropic interferences from those produced by natural events, we understand that the present study presents an unique opportunity for the comprehension of the steps involved in a stream community recovery after a natural disturbance. Furthermore, response velocity at community level remains one of the most valuable information regarding the understanding of community recovery process (Niemi et al., 1990; Detenbeck et al., 1992), e.g., how long does it take for composition, richness, and abundance to return to conditions prior to the disturbance? It is known that when a single event occurs, recovery can be achieved in days (Peterson and Bayley, 1993), weeks (Lonzarich et al., 1998), or even years (Detenbeck et al., 1992), depending on the colonization routes available (Carvalho and Uieda, 2006) and distance from a colonization source (like a preserved habitat, e.g. CenevivaBastos et al., 2012).

In Brazil, studies concerning recolonization of natural systems after disturbances are scarce, especially because information about richness and abundance of fauna and flora (or interactions between them) in a pre-disturbance period is usually absent. Notwithstanding, we had the opportunity to monitor the effects of a severe flash flood that occurred in a stream which had just been sampled by us for a food web study (M. Ceneviva-Bastos, pers. comm.). In this scenario, we aimed to investigate and compare the community attributes from the pre- and post-disturbance (post-disturbance I, II, and III) periods, and evaluate the existence of a temporal pattern in community structure that suggests return to conditions previous to the flash flood. 


\section{Material and Methods}

This study was conducted at the Córrego do Bagaço ( $20^{\circ} 24^{\prime} 08.5^{\prime \prime} \mathrm{S}$ and $\left.50^{\circ} 16^{\prime} 40.9^{\prime \prime} \mathrm{W}\right)$, a first order stream located at the Turvo river basin, northwestern São Paulo State. Samples were taken once before (PRED, 09/01/2007) and three times after the disturbance (POSD I, 03/16/2008; POSD II, 09/16/2008; POSD III, 08/15/2009). The interval among samples was determined based on in situ observations of the stream recovery process.

Samplings were conducted at a $60 \mathrm{~m}$ stream reach selected for its high variability of meso and microhabitats. Seven transects were positioned at each 10 meters to standardize different sampling methods, including the upstream and downstream limits. At each sample we measured dissolved oxygen (using a LT Lutron digital oximeter, DO - 5510 model), conductivity, $\mathrm{pH}$, turbidity, and temperature (with the Horiba electronic equipment, U-10 model). Stream width, depth, and flow were measured at each transect, the last measured at mid-channel and at the margins, with three replicates each. Substrate composition was visually estimated, along with the structural composition of the land-water ecotone and channel internal structure.

Planktonic organisms were sampled with a plankton net ( $45 \mu \mathrm{m}$ mesh size) at the pair transects, for four minutes. Benthic macroalgae composition and abundance (given by the percentage of substrate coverage by each morphospecies in relation to the total square area) were visually estimated in $l o c o$, with a sub aquatic viewer, before and after each odd transect, at its opposed sides (total of six samples), following methodology modified from Necchi (2004). The same procedure was used for aquatic macrophytes and samples of each producer morphospecies registered were taken to further identification at the laboratory. Benthic macroinvertebrates were sampled with a Surber net $(60 \mu \mathrm{m}$ mesh) by washing out the substrate for one minute at the odd transects and meiofauna was sampled using a PVC tube (10 centimeters diameter), buried five centimeters deep at the pair transects. Nektonic and epiphytic macroinvertebrates were sampled with a " $D$ " net (60 $\mu \mathrm{m}$ mesh size) passed along the 60 meters stream stretch, with total standardized effort of six minutes.

Fish were sampled with two electro-fishing passes $(220 \mathrm{~V}$ of alternated current, with $50-60 \mathrm{~Hz}$, 3.4-4.1 A e $1000 \mathrm{~W}$, with 50 minutes effort for each pass and with a 20 minutes interval between passes) along the entire stretch and macroinvertebrates caught with this methodology were also analyzed. All fish specimens were incorporated in the fish collection at the Departamento de Zoologia e Botânica da Universidade Estadual Paulista (DZSJRP), São José do Rio Preto, São Paulo State, Brazil.

All sampled specimens were anesthetized, fixed in formaldehyde, and conserved in ethanol $70 \%$ after 72 hours. Afterwards, they were identified up to genera or family level, using specific literature like Merritt and Cummins (1996), Costa et al. (2006) and Souza et al. (2007). Benthic macroalgae, aquatic macrophytes and periphyton were identified following Bicudo and Menezes (2006), Amaral et al. (2008) and Madigan et al. (2009). Specialists of each taxonomic group were consulted to confirm identifications made.

Planktonic organisms were counted in subsamples of collected material; larger individuals were counted using stereoscopic microscope, and smaller individuals were counted in Sedgwick-Rafter slide, using optical microscope. We used the same counting methodology for meiofauna invertebrates (Motta and Uieda, 2005). Benthic macroalgae, aquatic macrophytes and periphyton quantitative analysis were given by the percentage of substrate coverage by each morphospecies (Necchi, 2004), as mentioned above.

Richness and diversity values were obtained for each biotic group of each sampling period. Diversity was calculated by the Shannon-Wiener index (Magurran, 2004). Similarity of the aquatic biota between pre and pos-disturbance periods were examined by exploratory ordination, known as Non-Metric Multidimensional Scaling (NMDS) associated with Cluster Analysis, using quantitative and presence/absence Bray-Curtis similarity coefficients. Data for quantitative structure analysis were $\log (x+1)$ transformed. Analyses were conducted using the Primer 6.0 software (Clarke and Gorley, 2006). Presence and absence data were used for multivariate correlation analysis (Relate Analysis, Primer v. 6.0 software) in order to investigate taxonomic composition similarity of biota between pre and post-disturbance periods.

\section{Results}

Environmental variables demonstrated a series of changes in stream physical structure as a result of disturbance caused by the flash flood. The main changes were observed at the first period after the disturbance (POSD I), in which there was significant decrease in conductivity, as well as a fifteen-times-greater turbidity in comparison with pre-disturbance levels (Table 1). Increased water volume and flood erosive forces led stream width to become approximately 40 centimeters wider, while stream depth became 10 centimeters 
deeper. Mean current velocity increased almost $50 \%$. Stream bed composition was also modified, as detected by changes on the predominant substrate type. While prior to disturbance thinner particles like sand and silt were predominant (sand: $0.05-2.0 \mathrm{~mm}$; silt: $<0.05 \mathrm{~mm}$ ), posterior periods showed predominance of larger particles as boulder and rock (10.0-30.0 $\mathrm{mm}$ and $>30.0 \mathrm{~mm}$, respectively). Such rocks, however, were from upstream sliding, as a result from the high flow. The substrate that previously covered stream bed was almost completely removed after the flood.

Changes arising from disturbance could be better observed when comparing PRED and POSD I periods. With regards to vegetal cover composition (Table 2), for example, the high diversity registered in the pre-disturbance period was lost with the flood, with remains of only grassy riparian vegetation as the stream main cover in the POSD I period. In general, a progressive return of vegetal cover diversity along sampling periods could be observed, illustrated by similar composition (i.e. predominance of submerged-rooted vegetation, such as Echinodorus (Engelmann) and Ludwigia (Eames) between the PRED and POSD III periods.

A total amount of 13 producer genera was sampled in the pre-disturbance period (PRED, Table 3). In the next period (POSD I), composition was drastically altered with the removal of most producers by the flood. Only one algae and one macrophyte genera remained in the stream channel, evidencing the magnitude of the flash flood that led to a $96 \%$ loss of the initial vegetal cover. In the following period (POSD II), three genera that were present before the flood reestablished and other genera not registered until then, such as Spirogyra (Meunier) and Chaetophora, were also sampled. In the last sample (POSD III) other genera that originally composed stream vegetal cover, as the algae Oedogonium (Voekler) and the macrophyte Echinodorus (Engelmann), reestablished, the second being the most abundant in the pre-disturbance period. Notwithstanding, the percentage of algae and macrophyte cover in the POSD III period demonstrated a decrease of nearly $53 \%$ of original stream cover despite the observed recolonization.

Table 1. Mean values of environmental variables sampled in the sampling periods (pre-disturbance - PRED; postdisturbance I - POSD I; post-disturbance II - POSD II; and post-disturbance III - POSD III).

\begin{tabular}{lcccc}
\hline \multicolumn{1}{c}{ Variables } & PRED & POSD I & POSD II & POSD III \\
\hline Dissolved oxygen $(\mathrm{mg} / \mathrm{l})$ & 5 & 6.3 & 8.2 & 12.8 \\
Conductivity $(\mu \mathrm{S} / \mathrm{cm})$ & 20 & 0.08 & 0.047 & 0.036 \\
$\mathrm{pH}$ & 7.46 & 8.4 & 7.29 & 6.52 \\
Turbidity $(\mathrm{NTU})$ & 2 & 29 & 3 & 5 \\
Temperature $\left({ }^{\circ} \mathrm{C}\right)$ & 23.2 & 23.2 & 21.6 & 19.7 \\
Width $(\mathrm{m})$ & 1.74 & 2.12 & 1.86 & 1.51 \\
Depth $(\mathrm{cm})$ & 14.56 & 25.09 & 0.17 & 18.57 \\
Current velocity $(\mathrm{m} / \mathrm{s})$ & 0.09 & boulder/rock & silt & 0.29 \\
Predominant substrate type & sand/silt & & & boulder \\
\hline
\end{tabular}

Table 2. Predominant vegetal cover composition between transects (form T1 to T7), in the sampled periods (pre-disturbance - PRED; post-disturbance I - POSD I; post-disturbance II - POSD II; and post-disturbance III - POSD III).

\begin{tabular}{|c|c|c|c|c|c|c|}
\hline & T1-T2 & T2-T3 & T3-T4 & T4-T5 & T5-T6 & T6-T7 \\
\hline PRED & $\begin{array}{c}\text { Rooted } \\
\text { submerged } \\
\text { vegetation }\end{array}$ & $\begin{array}{c}\text { Rooted } \\
\text { submerged } \\
\text { and emergent } \\
\text { vegetation }\end{array}$ & $\begin{array}{l}\text { Rooted } \\
\text { submerged } \\
\text { vegetation }\end{array}$ & $\begin{array}{c}\text { Rooted } \\
\text { emerged } \\
\text { vegetation }\end{array}$ & $\begin{array}{l}\text { Floating } \\
\text { vegetation; } \\
\text { rooted } \\
\text { emergent and } \\
\text { submerged } \\
\text { vegetation }\end{array}$ & $\begin{array}{c}\text { Rooted } \\
\text { submerged } \\
\text { and emergent } \\
\text { vegetation }\end{array}$ \\
\hline POSD I & $\begin{array}{c}\text { Grass in } \\
\text { contact with } \\
\text { water }\end{array}$ & $\begin{array}{c}\text { Grasses; grass } \\
\text { in contact with } \\
\text { water }\end{array}$ & Adhered algae & $\begin{array}{c}\text { Grasses; grass } \\
\text { in contact with } \\
\text { water }\end{array}$ & $\begin{array}{l}\text { Grasses; grass } \\
\text { in contact with } \\
\text { water }\end{array}$ & $\begin{array}{c}\text { Grasses; grass } \\
\text { in contact with } \\
\text { water }\end{array}$ \\
\hline POSD II & Floating algae & $\begin{array}{l}\text { Rooted } \\
\text { submerged } \\
\text { vegetation }\end{array}$ & $\begin{array}{l}\text { Rooted } \\
\text { submerged } \\
\text { vegetation }\end{array}$ & $\begin{array}{l}\text { Rooted } \\
\text { submerged } \\
\text { vegetation }\end{array}$ & $\begin{array}{l}\text { Floating } \\
\text { vegetation }\end{array}$ & $\begin{array}{c}\text { Rooted } \\
\text { submerged } \\
\text { vegetation; } \\
\text { adhered and } \\
\text { floating algae }\end{array}$ \\
\hline POSD III & $\begin{array}{c}\text { Rooted } \\
\text { submerged } \\
\text { vegetation }\end{array}$ & $\begin{array}{c}\text { Rooted } \\
\text { submerged } \\
\text { vegetation }\end{array}$ & $\begin{array}{c}\text { Rooted } \\
\text { submerged } \\
\text { vegetation }\end{array}$ & Grasses & $\begin{array}{c}\text { Rooted } \\
\text { submerged } \\
\text { vegetation }\end{array}$ & $\begin{array}{c}\text { Rooted } \\
\text { submerged } \\
\text { vegetation }\end{array}$ \\
\hline
\end{tabular}


Table 3. Richness and abundance of each taxa sampled in the studied periods (pre-disturbance - PRED; post-disturbance I - POSD I; post-disturbance II - POSD II; and post-disturbance III - POSD III). Algae and macrophytes abundance was determined through visual estimation of hole reach coverage percentage.

\begin{tabular}{|c|c|c|c|c|c|}
\hline Taxa & & PRED & POSD I & POSD II & POSD III \\
\hline \multirow{19}{*}{ Algae and macrophytes } & Oedogonium & 2.5 & 1.0 & - & 1.3 \\
\hline & Zygnema & 1.7 & - & - & - \\
\hline & Spirogyra & - & - & 5.0 & - \\
\hline & Chaetophora & - & - & 15.0 & - \\
\hline & Heteranthera & 4.2 & - & - & - \\
\hline & Echinodorus & 29.2 & - & - & 6 \\
\hline & Diodia & 2.5 & - & - & - \\
\hline & Mikania & 1.7 & - & - & - \\
\hline & Myriophyllum & 4.2 & - & 8.3 & 9.2 \\
\hline & Brachiaria & 6.7 & 1.7 & - & - \\
\hline & Centella & 4.2 & - & - & - \\
\hline & Ranunculus & 4.2 & - & 6.7 & 4.2 \\
\hline & Ludwigia & 6.7 & - & 1.7 & 8.8 \\
\hline & Cyperus & 2.5 & - & - & - \\
\hline & Baccharidastrum & 0.8 & - & - & - \\
\hline & Formidium & - & - & - & 5.5 \\
\hline & Filamentous bacteria & - & - & - & 2.5 \\
\hline & Abundance (cover percentage) & 70.8 & 2.7 & 36.7 & 36.2 \\
\hline & Richness & 13 & 2 & 5 & 7 \\
\hline \multirow{40}{*}{ Macroinvertebrates } & Anacroneuria & 11 & 1 & 12 & 6 \\
\hline & Americabaetis & 97 & 50 & 163 & 222 \\
\hline & Apobaetis & 4 & - & 10 & - \\
\hline & Cryptonympha & 1 & - & 4 & - \\
\hline & Callibaetis & - & - & 43 & - \\
\hline & Waltzoyphius & - & - & - & 43 \\
\hline & Zelusia & - & - & - & 3 \\
\hline & Aturbina & - & - & 5 & - \\
\hline & Traverhyphes & 11 & 4 & 16 & 122 \\
\hline & Tricorythodes & 3 & - & 1 & 5 \\
\hline & Tricorythopsis & 1 & - & - & - \\
\hline & Caenis & - & - & 1 & 5 \\
\hline & Farrodes & 98 & - & 11 & 66 \\
\hline & Smicridea & 18 & 1 & 20 & 109 \\
\hline & Leptonema & 143 & 44 & 210 & 87 \\
\hline & Macronema & 8 & - & 7 & 46 \\
\hline & Macrostemum & 16 & 1 & 3 & 16 \\
\hline & Hydroptila & - & - & - & 1 \\
\hline & Cyrnellus & - & 1 & - & 10 \\
\hline & Chimarra & 2 & 1 & 1 & - \\
\hline & Marilia & 6 & 7 & 20 & 2 \\
\hline & Helicopsyche & - & - & 60 & 31 \\
\hline & Cladopelma & 3 & - & - & 1 \\
\hline & Tanytarsus & 3 & - & - & 17 \\
\hline & Polypedillum (Tripodura)* & 1 & 7 & 2 & 17 \\
\hline & Chironominae & 8 & - & - & - \\
\hline & Onconeura & 102 & 4 & 2 & 13 \\
\hline & Cricotopus & 19 & 5 & 20 & 207 \\
\hline & Rheotanytarsus & 4 & - & 2 & 18 \\
\hline & Parachironomus & 1 & - & 2 & 12 \\
\hline & Endotribelos & 1 & - & - & 3 \\
\hline & Thienemanniela & 10 & - & 1 & 5 \\
\hline & Parametriocnemus & 33 & - & 5 & 19 \\
\hline & Corynoneura & 1 & - & - & 2 \\
\hline & Nanocladius & 3 & - & 3 & 33 \\
\hline & Stenochironomus & 1 & - & - & 1 \\
\hline & Pentaneura & 15 & - & 5 & 56 \\
\hline & Ablabesmyia (Karelia)* & 4 & - & 4 & 18 \\
\hline & Labrundinia & 1 & - & - & - \\
\hline & Tanypodinae & 2 & - & - & - \\
\hline
\end{tabular}

${ }^{*}$ Trivino-Strixino and Strixino (1994). ${ }^{* *}$ Passos et al. (2007). 
Table 3. Continued...

\begin{tabular}{|c|c|c|c|c|c|}
\hline Taxa & & PRED & POSD I & POSD II & POSD III \\
\hline & Denopelopia & 1 & - & - & - \\
\hline & Polypedillum & - & 7 & 35 & - \\
\hline & Polypedillum gen. Falax & - & 1 & 1 & 1 \\
\hline & Fissimentum & - & 1 & - & - \\
\hline & Beardius & - & - & 1 & 10 \\
\hline & Marnischia & - & - & 1 & - \\
\hline & Dicrotendipes & - & - & 1 & 2 \\
\hline & Gr. Thienemannimyia* & - & - & 4 & 6 \\
\hline & Clinotanypus & - & - & - & 2 \\
\hline & Tanytarsini genus $\mathrm{B}^{*}$ & - & - & - & 2 \\
\hline & Lauterborniella & - & - & - & 2 \\
\hline & Phaenoespectra & - & - & - & 6 \\
\hline & Pseudochironomus & - & - & - & 6 \\
\hline & Chironomus gr. Riparius* & - & - & - & 1 \\
\hline & Nimbocera & - & - & - & 1 \\
\hline & Cladomyia & - & - & - & 1 \\
\hline & Coelotanypus & - & - & - & 20 \\
\hline & Larsia & - & - & - & 1 \\
\hline & Tabanidae & - & - & - & 1 \\
\hline & Procladius & - & - & - & 2 \\
\hline & Simulium & 967 & 145 & 13 & 101 \\
\hline & Empididae & 1 & - & - & - \\
\hline & Tipulidae & 2 & - & - & 1 \\
\hline & Tabanidae & - & - & 3 & 4 \\
\hline & Atrichopogon & - & - & 4 & - \\
\hline & Ceratopogonidae & - & - & 1 & - \\
\hline & Dixidae & 1 & - & 3 & 11 \\
\hline & Limnocoris & 191 & 43 & 225 & 348 \\
\hline & Ambrysus & 2 & 2 & 1 & 10 \\
\hline & Halobatopsis & 4 & - & 21 & - \\
\hline & Gerridae & - & - & - & 1 \\
\hline & Rheumatobates & - & - & - & 1 \\
\hline & Brachymetra & - & - & - & 1 \\
\hline & Aquarius & - & - & - & 2 \\
\hline & Belostoma & 1 & 2 & - & 2 \\
\hline & Rhagovelia & - & - & 6 & 7 \\
\hline & Platyvelia & - & - & - & 2 \\
\hline & Microvelia & 1 & - & - & - \\
\hline & Martarega & - & 1 & - & - \\
\hline & Elmidae & 46 & 2 & 15 & - \\
\hline & Elmidae larvae $\mathrm{B}^{* *}$ & - & - & - & 5 \\
\hline & Elmidae larvae $C^{* *}$ & - & - & 4 & 4 \\
\hline & Elmidae larvae $D^{* *}$ & - & - & 1 & - \\
\hline & Elmidae larvae $E^{* *}$ & - & - & 2 & 2 \\
\hline & Elminae & - & - & 3 & 1 \\
\hline & Hexacylloepus sp1 & - & - & 7 & 9 \\
\hline & Hexacylloepus sp2 & - & - & 7 & - \\
\hline & Heterelmis & - & - & 1 & 1 \\
\hline & Phanocerus & - & - & - & 1 \\
\hline & Neoelmis & - & - & - & 2 \\
\hline & Macrelmis & - & - & 2 & 1 \\
\hline & Xenelmis & - & - & - & 1 \\
\hline & Mycrocylloepus & - & - & - & 1 \\
\hline & Curculionidae & - & - & - & 1 \\
\hline & Dryopidae & - & - & - & 1 \\
\hline & Coleoptera & - & - & 1 & 1 \\
\hline & Hydrophilidae & - & 1 & 2 & 1 \\
\hline & Girinidae & - & - & 2 & 1 \\
\hline & Hydrochidae & 1 & - & - & - \\
\hline & Dytiscidae & - & - & 2 & 1 \\
\hline & Hydraenidae & 1 & - & - & - \\
\hline
\end{tabular}

*Trivino-Strixino and Strixino (1994). ${ }^{* *}$ Passos et al. (2007). 
Table 3. Continued...

\begin{tabular}{|c|c|c|c|c|c|}
\hline \multicolumn{2}{|c|}{ Taxa } & \multirow{2}{*}{$\begin{array}{c}\text { PRED } \\
1\end{array}$} & \multirow{2}{*}{$\begin{array}{c}\text { POSD I } \\
-\end{array}$} & \multirow{2}{*}{ POSD II } & \multirow{2}{*}{$\frac{\text { POSD III }}{-}$} \\
\hline & Scirtidae & & & & \\
\hline & Noteridae & - & - & - & 2 \\
\hline & Lutrochidae & - & - & - & 1 \\
\hline & Pyralidae sp1 & 1 & - & - & 30 \\
\hline & Pyralidae sp2 & 1 & - & - & - \\
\hline & Rhionaeshna & 6 & - & - & - \\
\hline & Neuraeshna & 1 & - & - & - \\
\hline & Hetaerina & 45 & 4 & 7 & 6 \\
\hline & Libellulidae & 2 & - & - & 13 \\
\hline & Dasythemis & - & - & - & 2 \\
\hline & Brechmorhoga & 55 & - & 8 & 6 \\
\hline & Elasmothemis & 21 & - & 18 & 22 \\
\hline & Planiplax & 20 & - & 7 & - \\
\hline & Perithemis & - & 2 & - & 1 \\
\hline & Anatya & 3 & - & 2 & 5 \\
\hline & Erythrodiplax & 7 & - & 3 & - \\
\hline & Tramea & 9 & - & 9 & - \\
\hline & Oligoclada & - & - & 1 & - \\
\hline & Macrothemis & 6 & - & 10 & 4 \\
\hline & Diastops & 1 & - & - & - \\
\hline & Gomphoides & 5 & - & - & - \\
\hline & Phyllocycla & 25 & 1 & 8 & 19 \\
\hline & Progomphus & 6 & - & 8 & - \\
\hline & Coenagrionidae & 29 & 2 & 12 & 34 \\
\hline & Argia & 8 & - & - & 19 \\
\hline & Macrobrachium & 26 & 3 & 10 & 37 \\
\hline & Dilocarcinus & - & 9 & - & - \\
\hline & Biomphalaria & - & - & - & 119 \\
\hline & Oligochaeta & - & - & 11 & 7 \\
\hline & Annelida & - & - & - & 2 \\
\hline & Hyrudinea & - & - & - & 1 \\
\hline & Collembola & - & - & - & 3 \\
\hline & Nematoda & - & - & 1 & - \\
\hline & Abundance & 2128 & 352 & 1117 & 2118 \\
\hline & Richness & 66 & 28 & 70 & 97 \\
\hline \multirow{26}{*}{ Fish } & Parodon nasus & 2 & 7 & 7 & 5 \\
\hline & Staindachnerina insculpta & 7 & 1 & 4 & 2 \\
\hline & Prochilodus lineatus & 3 & - & - & - \\
\hline & Characidium zebra & 16 & 6 & 12 & 20 \\
\hline & Astyanax altiparanae & 50 & 18 & 15 & 26 \\
\hline & Astyanax fasciatus & 13 & - & 4 & 4 \\
\hline & Hemigrammus marginatus & 15 & 5 & 1 & 11 \\
\hline & Moenkhausia sanctaefilomenae & 1 & - & - & - \\
\hline & Oligosarcus pintoi & 1 & 1 & - & - \\
\hline & Piabina argenta & 23 & 8 & 8 & 2 \\
\hline & Salminus hilarii & 1 & - & - & - \\
\hline & Serrapinnus heterodon & 36 & - & - & 3 \\
\hline & Serrapinnus notomelas & 22 & 1 & 10 & 33 \\
\hline & Hoplias malabaricus & 3 & - & - & - \\
\hline & Aspidoras fuscoguttatus & 14 & 3 & 14 & 10 \\
\hline & Corydoras aeneus & - & 2 & - & 2 \\
\hline & Hypostomus ancistroides & 5 & 28 & 39 & 18 \\
\hline & Hypostomus nigromaculatus & 3 & 6 & 14 & 6 \\
\hline & Gymnotus sylvius & 31 & 16 & 5 & 22 \\
\hline & Cichlasoma paranaense & - & - & 2 & - \\
\hline & Crenicichla britskii & 8 & - & 1 & 12 \\
\hline & Satanoperca pappaterra & 1 & - & - & - \\
\hline & Geophagus brasiliensis & - & - & - & 5 \\
\hline & Cyphocharax modestus & - & - & - & 1 \\
\hline & Abundance & 255 & 102 & 136 & 182 \\
\hline & Richness & 20 & 13 & 14 & 17 \\
\hline
\end{tabular}

${ }^{*}$ Trivino-Strixino and Strixino (1994). ${ }^{* *}$ Passos et al. (2007). 
Initial macroinvertebrate community presented 66 morphospecies (Table 3), being reduced to $42 \%$ in the first period after the flood. However, changes in total abundance were more expressive, with 2,128 specimens before the flood and only 352 individuals in the POSD I period (83\% of abundance loss). Nevertheless, macroinvertebrate abundance showed a growing increase in the next periods, achieving a total amount of 2,118 organisms in the last sample (POSD III). Several invertebrate genera reestablished, such as two Ephemeroptera, one Trichoptera, 14 Diptera, one Lepidoptera, and five Odonata. Additionally, 53 new genera colonized the stream and were present in the last sampling period (POSD III).

Ichthyofauna richness also followed this pattern, with higher values in the PRED period, lowest in the POSD I period, and further gradual increase (Table 3). Of the initial 20 fish species sampled, only thirteen were caught after the flash flood (40\% of abundance loss). Prochilodus lineatus (Valenciennes,
1837), Moenkhausia sanctaeflomenae (Steindachner, 1907), Salminus hilarii Valenciennes, 1850, Hoplias malabaricus (Bloch, 1794), and Satanoperca pappaterra (Heckel, 1840) were no longer sampled in the next periods. However, ichthyofauna recolonization counted with three species sampled in POSD III that had not been registered in predisturbance period, Corydoras aeneus (Gill, 1858), Geophagus brasiliensis (Quoy and Gaimard, 1824), and Cyphocharax modestus (Fernández-Yépez, 1948).

Richness and diversity values for aquatic biota of each sampling period are presented in Table 4. Shannon-Wiener index values indicate that, despite changes in composition, the relation between species richness and abundance tended to reestablish towards the pre-flood period. Indeed, the Nonmetric Multidimensional Scaling (NMDS) results showed 55\% similarity among PRED, POSD II, and POSD III regarding both abundance and composition (Figure 1). However, the relation among these periods is different. In

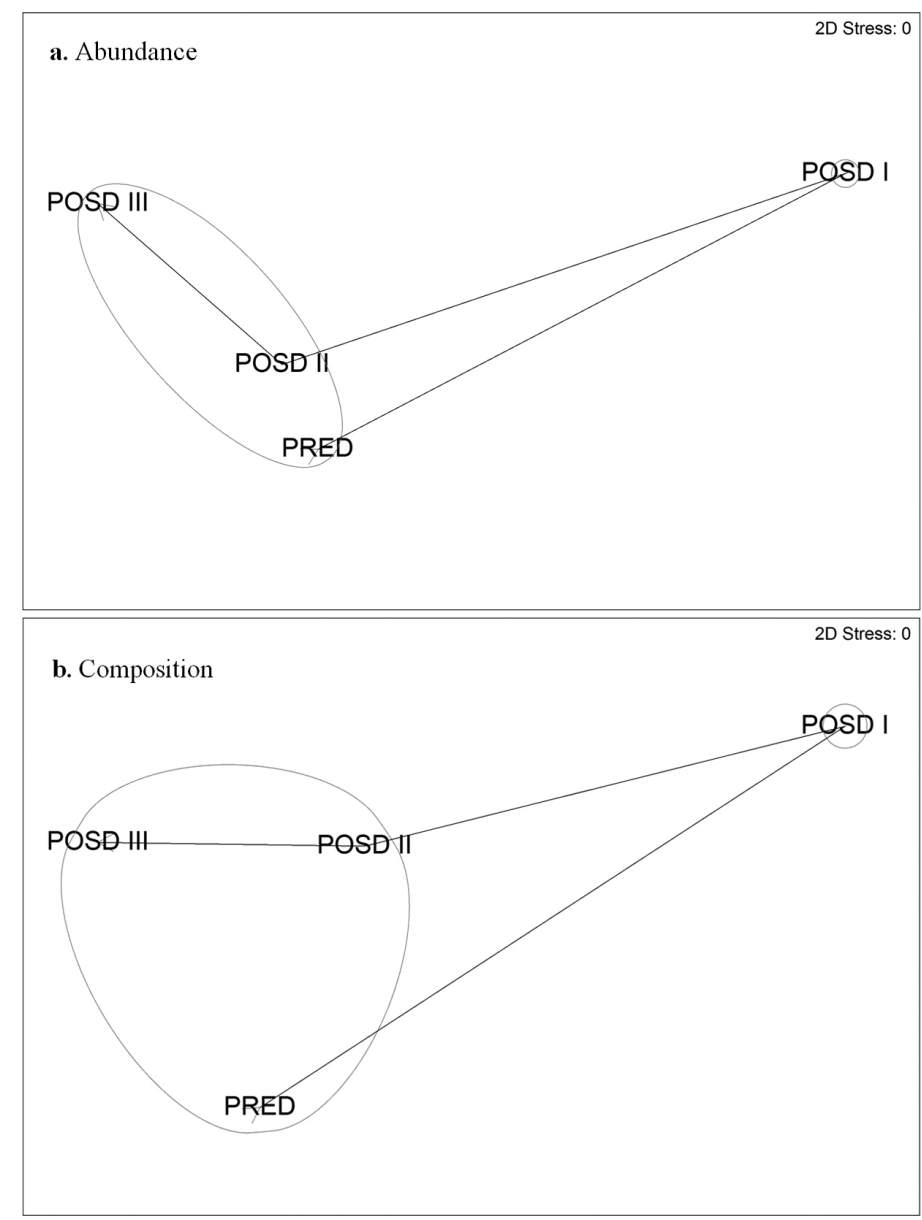

Figure 1. Non-Metric Multidimensional Scaling associated with Cluster Analysis evidencing groups formed from the pre-disturbance (PRED) and post-disturbance I, II, and III (POSD I, POSD II, and POSD III, respectively) periods, using Bray-Curtis index for abundance (a) and composition (b). Circles represent groups with 55\% similarity. Lines indicate temporal sequence and direction of the stream community recovery process. 
Table 4. Richness $(S)$ and Shannon-Wiener diversity index $\left(\mathrm{H}^{\prime}\right)$ values for aquatic biota in the sampled periods (predisturbance - PRED; post-disturbance I - POSD I; post-disturbance II - POSD II; and post-disturbance III - POSD III).

\begin{tabular}{|c|c|c|c|c|c|c|c|c|}
\hline \multirow[t]{2}{*}{ Taxa } & \multicolumn{2}{|c|}{ PRED } & \multicolumn{2}{|c|}{ POSD I } & \multicolumn{2}{|c|}{ POSD II } & \multicolumn{2}{|c|}{ POSD III } \\
\hline & $\mathbf{S}$ & $\mathrm{H}^{\prime}$ & $\mathbf{S}$ & $\mathrm{H}^{\prime}$ & $\mathbf{s}$ & $\mathrm{H}^{\prime}$ & $\mathbf{S}$ & $\mathrm{H}^{\prime}$ \\
\hline Algae and macrophytes & 13 & 0.89 & 2 & 0.29 & 5 & 0.62 & 7 & 0.78 \\
\hline Macroinvertebrates & 66 & 1.03 & 28 & 0.89 & 70 & 1.28 & 97 & 1.44 \\
\hline Fish & 20 & 1.08 & 13 & 0.93 & 14 & 0.98 & 17 & 1.07 \\
\hline Total & 99 & 3.00 & 43 & 2.11 & 89 & 2.88 & 121 & 3.29 \\
\hline
\end{tabular}

both quantitative and qualitative analysis, the first subsequent period after disturbance (POSD I) is significantly isolated from the others, which indicates a greater community structure disparity among the period right after the flood and the subsequent ones.

Multivariate correlation analysis (Relate Analysis) indicated a greater correlation between macroinvertebrates and algae $(\mathrm{Rho}=0.7, \mathrm{P}<0.05)$. The correlation between macroinvertebrates and fish (Rho $=-0.4, \mathrm{P}>0.05)$ and between fish and algae (Rho $=-0.09, \mathrm{P}>0.05)$ were not significant. Such results demonstrate the narrow relation between invertebrates and algae recolonization dynamics.

\section{Discussion}

Data herein obtained had evidenced that flash floods can act as a driving force to modify stream physical structure and biota, as observed in many other studies concerning stream recolonization (Pickett and White, 1985; Lytle, 2000; Yount and Niemi, 1990; Franssen et al., 2006). The time necessary for community recovery, tending to the return to conditions prior to disturbance including the reestablishment of richness and diversity, was notably slow (almost two years), when compared to other studies in which recovery was reached in months (Fisher et al., 1982), weeks (Lonzarich et al., 1998), or even days (Peterson and Bayley, 1993). In a review of 411 cases of ichthyofauna recolonization conducted by Detenbeck et al. (1992), 70\% of composition, richness, and total density recovered in less than a year. However, it usually takes from few months to one or more years to complete recovery (Fisher et al., 1982).

The relatively and notably long-term recovery of the community assessed in the studied stream can be explained by the drastic changes in substrate composition (Table 1), since substrate type is often considered one of the physical factors more intimately associated to disturbance consequences (Pickett and White, 1985). The increase in channel velocity, depth, and width were changes that evidenced flood high magnitude. Thus, the greater water volume, higher velocity, and flood erosive forces might have caused a substrate scouring and sediment transport (Matthews, 1998; Lytle, 2000), with remains of only naked rocks on stream bed, as observed in POSD I period. Indeed, substratum removal, along with the removal of algae and macroinvertebrates, are some of the most common consequences of erosive floods (Matthews, 1998; Grimm and Fisher, 1989).

The combination of reduced diversity and absence of appropriate substrate might have led to changes on the establishment and fixing of algae and macrophytes. During floods, the high current velocity may drastically alter aquatic plant communities through mechanic removal of vegetal cover and thinner sediment (Henry et al., 1994). In studies of flash floods effects on the substrate and aquatic vegetation, Henry et al. (1994, 1996) found significant changes on substrate grain-size. During the flash flood, water flow removed fine sediments, bringing larger and coarse particles such as gravels and boulders (Henry et al., 1996). In both studies, vegetal cover decrease was registered after the flood, mainly where greater grain-size sediments predominated; also, many species that occurred before the disturbance were no longer sampled. Grimm and Fisher (1989) observed low algae resistance against flood events capable of washing the substrate out, being also observed by Maltchik and Pedro (2001) for macrophytes. Maltchik and Pedro (2001) reported a positive correlation between flood magnitude and abundance decrease of aquatic plants. Substrate modification and consequent vegetal cover reduction herein observed (96\% loss, Table 3) corroborate the results of the studies mentioned above, and, as suggested by Henry et al. (1996), the long-term recolonization might have been an effect of the removal of almost all vegetal elements.

Many studies have also demonstrated the destructive effect of floods on macroinvertebrate communities, drastically reducing abundance and 
diversity of these organisms (Grimm and Fisher, 1989; Nislow et al., 2002; Lee and Bae, 2011; Mundahl and Hunt, 2011). The high decrease of macroinvertebrate abundance observed at Córrego do Bagaço stream ( $83 \%$ abundance loss) is comparable to those related after floods at Sycamore Creek, Arizona (86\% loss, Gray, 1981; 96\% loss, Fisher et al., 1982), Tesuque Creek, New Mexico (94\% loss, Molles Junior, 1985), and North Fork Cave Creek, Arizona (95\% loss, Lytle, 2000). The decrease of macroinvertebrates richness and diversity from Córrego do Bagaço might have been caused by the substrate scouring and consequent removal of organisms by the flood (Fisher et al., 1982; Matthews, 1998; Death, 2010), which may be carried downstream along with FPOM by substrate movement and be subject to desiccation (Lytle, 2000). In a study of invertebrate drift, Gibbins et al. (2007) also found a positive relation between the loss of benthic organisms and sediment transport rate.

Despite the severe reduction of macroinvertebrates richness and diversity in the POSD I sampling period, recolonization was progressive (see Table 3 ). According to Williams and Hynes (1976) benthos recolonization evolves four main mechanisms: drift, upstream migration, vertical movement from deeper substrate refugia, and adult oviposition by aerial sources. Due to flood magnitude and to increased current velocity, macroinvertebrate recolonization by upstream migration is less probable. Additionally, sediment transport and scouring of almost the entire stream bed hampers recolonization from substrate refugia. Thus, drift and post-flood adult oviposition might have been the most important recolonization routes for the recovery of macroinvertebrate biota. The importance of these two mechanisms is largely known in studies concerning the dynamics of stream macroinvertebrates (Williams and Hynes, 1976; Fisher et al., 1982; Lytle, 2000; Carvalho and Uieda, 2006; Death, 2010).

As for other groups of aquatic biota, fish richness and abundance were reduced after the flood, although alterations were milder when compared to those observed for algae, macrophytes, and macroinvertebrates communities. Pires et al. (2008) found that fish richness and abundance changed little after a severe flash flood, and changes that were observed were mostly on cyprinid species. Jurajda et al. (2006) registered loss of eight fish species after a flood, with reduction also in their abundance, though posterior recolonization showed an occurrence of four species that were not present prior to the disturbance. Similar results were found at the studied stream (see Table 3). Changes in ichthyofauna might be a result of stream bed scouring, which can modify micro-habitat availability for fish through the removal of macrophyte cover, for instance, as it can also destroy nests by mechanical forces due to sediment lifting (Matthews, 1998). Matthews (1998) also highlighted other flood effects that can disturb fish assemblage, as scouring of algae and macroinvertebrates can alter fish food resources, modifying stream trophic structure, and the high turbidity itself and channel erosion may directly affect reproductive success and fish growing (Matthews, 1998). Notwithstanding, changes observed herein are unlikely to be related to food resource availability, since most species sampled are generalists/opportunists (as in CenevivaBastos et al., 2010) regarding feeding habits (personal observation). Ichthyofauna recolonization and recovery might have occurred by upstream dispersal, as the study site is placed near a larger river that could act as a stream recolonization source.

Despite the remarkably slow recolonization observed, along with the immediate negative flood effects on richness and abundance, it was possible to observe that overall community structure did tend to return to previous conditions, evidencing community resilience despite some species turnover. Indeed, many studies have shown the role of disturbance in shaping stream communities (Resh et al., 1988; Lake, 2000) and stream ecology have been providing a great body of evidence that support the role of stochastic forces in controlling community structure other than deterministic biotic interactions, such as competition for example (Chase, 2007; Death, 2010).

Streams are known to be relatively unstable environments for being subject to disturbances that directly affect flow regime and environmental conditions, and stream biota appear to have life-history traits that can buffer local populations against adverse conditions (Winemiller et al., 2010). Notwithstanding, some disturbances as observed herein can greatly reduce richness and abundance of local individuals or even deplete them from certain habitat patches, so community persistence through time depends on recruitment from outside sources (Winemiller et al., 2010), and recolonization depends on availability, size (Lancaster, 2000), and quality of refugia (Matthaei et al., 2000). In this context, anthropic impacts can limit quantity and quality of refugia 
through habitat homogenization in behalf of complete deforested and silted stream conditions (Casatti et al., 2009), a very common consequence of land use for livestock grazing at the studied region. This whole scenario can explain both the flood magnitude, which could have had a much lower impact in streams with a preserved riparian buffer zone, and the slow recolonization observed.

\section{Conclusion}

Flash floods are known by its capacity of altering stream communities regarding both physical structure and aquatic biota. Increases of water volume and flow velocity may cause sediment lifting and substrate scouring, which are some of the most frequent consequences after these events. These physical alterations were observed at the studied stream, followed by changes in composition and abundance of vegetal cover, macroinvertebrate, and fish assemblages. Changes were characterized by an abrupt initial reduction of species richness and abundance, with a gradually slow recovery during post-flood periods. After the flood, biota recolonization might have included external sources (e.g., laying eggs by adult insects) and/or internal sources (fish upstream dispersal), highlighting the different pathways through which a stream community can restructure after a natural event. Thus, a stream community progressive recovery trend could be herein observed, supported by the return of aquatic biota abundances towards its original values. On the other hand, species composition showed significant differences among sampling periods, demonstrating that species groups might be characteristic of each temporal unit, although community composition in the last samples was more similar to the stream original condition one. Finally, understanding the dynamics of stream natural recovery process can bring important insights on forecasting possible consequences of anthropic interferences, as anthropogenic disturbances can also lead to stream biota depletion. The modifications observed in the studied stream by the result of a flash flood could be much less expressive if the riparian vegetation was present, buffering stream habitat patches and biota against such a strong disturbance. Moreover, the anthropic influence indirectly affected stream recovery, as the absence of riparian vegetation is related to the presence of livestock and lack of any management actions towards stream conservation.

\section{Acknowledgements}

We thank CNPq (PIBIC/UNESP 10845) and FAPESP for funding (process number 06/04937-
3), Cláudio G. Froehlich, Luís O. I. de Souza (in memorian), Orlando Necchi Jr., Luiz H. Z. Branco, Francisco Langeani, Maria Stela M. C. Noll, and Fernando B. Noll for confirming taxonomic identifications, and Cristiane P. Ferreira for analysis support.

\section{References}

AMARAL, MCE., BITTRICH, V., FARIA AD., ANDERSON, LO. and AONA, LYS. 2008. Guia de campo para plantas aquáticas e palustres do Estado de São Paulo. Ribeirão Preto: Holos Editora. 449 p.

BARRAT-SEGRETAIN, MH. and AMOROS, C. 1995. Recovery of riverine vegetation after experimental disturbance: a field test of the patch dynamics concept. Hydrobiologia, vol. 316, p. 91-101.

BICUDO, CEM. and MENEZES, M. 2006. Gêneros de algas continentais do Brasil - chave para identificação e descriçōes. RiMa: São Carlos. 489 p.

CARVALHO, EM. and UIEDA, VS. 2006. Colonization routes of benthic macroinvertebrates in a stream in southeast Brazil. Acta Limnologica Brasiliensia, vol. 18 , no. 4, p. 367-376.

CASATTI, L., FERREIRA, CP. and CARVALHO, FR. 2009. Grass-dominated stream sites exhibit low fish species diversity and dominance by guppies: an assessment of two tropical pasture river basins. Hydrobiologia, vol. 632, p. 273-283. http://dx.doi. org/10.1007/s10750-009-9849-y

CELlOT, B., MOUILlOT, F. and HENRY, CP. 1998. Flood drift and propagule bank of aquatic macrophytes in a riverine wetland. Journal of Vegetation Science, vol. 9, p. 631-640. http://dx.doi. org/10.2307/3237281

CENEVIVA-BASTOS, M., CASATTI, L. and ROSSAFERES, DC. 2010. Meso and microhabitat analysis and feeding habits of small nektonic characins (Teleostei: Characiformes) in Neotropical streams. Zoologia, vol. 27, no. 2, p. 191-200. http://dx.doi. org/10.1590/S1984-46702010000200006

CENEVIVA-BASTOS, M., CASATTI, L. and UIEDA, VS. 2012. Can seasonal differences influence food web structure on preserved habitats? Responses from two Brazilian streams. Community Ecology, vol. 13, no. 2, p. 243-252. http://dx.doi.org/10.1556/ ComEc.13.2012.2.15

CHASE, JM. 2007. Drought mediates the importance of stochastic community assembly. Proceedings of the National Academy of Sciences of the United States of America, vol. 104, p. 17430-17434. PMid:17942690 PMCid:PMC2077273.

ClARKE, KR. and GORLEY, RN. 2006. Primer v6: user manual/tutorial. Plymouth: Plymouth Marine Laboratory. 
COSTA, C., IDE, S. and SIMONKA, CE. 2006. Insetos imaturos, metamorfose e identificação. Ribeirão Preto: Holos Editora. 249 p.

DEATH, RG. 2010. Disturbance and riverine benthic communities: what has it contributed to general ecological theory? River Research and Applications, vol. 26, p. 15-25. http://dx.doi.org/10.1002/rra.1302

DETENBECK, NE., DEVORE, PW., NIEMI, GJ. and LIMA, A. 1992. Recovery of temperate-stream fish communities from disturbance: a review of case studies and synthesis of theory. Environmental Management, vol. 16, no. 1, p. 33-53. http://dx.doi. org/10.1007/BF02393907

FISHER, SG., GRAY, LJ., GRIMM, NB. and BUSCH, DE. 1982. Temporal succession in a desert stream ecosystem following flash flooding. Ecological Monographs, vol. 52, no. 1, p. 93-110. http://dx.doi. org/10.2307/2937346

FRANSSEN, NR., GIDO, KB., GUY, CS., TRIPE, JA., SHRANK, S., STRAKOSH, TR., BERTRAND, KN., FRANSSEN, CM., KRISTEN, LP. and PAUKERT, CRP. 2006. Effects of foods on fish assemblages in an intermittent prairie stream. Freshwater Biology, vol. 51, p. 2072-2086. http:// dx.doi.org/10.1111/j.1365-2427.2006.01640.x

GIBBINS, C., VERICA, D. and BATALLA, RJ. 2007. When is stream invertebrate drift catastrophic? The role of hydraulics and sediment transport in initiating drift during flood events. Freshwater Biology, vol. 52, p. 2369-2384. http://dx.doi.org/10.1111/j.13652427.2007.01858.x

GRAY, LJ. 1981. Species composition and life histories of aquatic insects in a lowland sonoran desert stream. American Midland Naturalist, vol. 106, no. 2, p. 229242. http://dx.doi.org/10.2307/2425159

GRIMM, NB. and FISHER, SG. 1989. Stability of Periphyton and Macroinvertebrates to Disturbance by Flash Floods in a Desert Stream. Journal of the North American Benthological Society, vol. 8, no. 4, p. 293-307. http://dx.doi.org/10.2307/1467493

HENRY, CP., BORNETTE, G. and AMOROS, C. 1994. Differential effects of floods on the aquatic vegetation of braided channels of the Rhône River. Journal of North American Benthological Society, vol. 13, no. 4, p. 439-467. http://dx.doi.org/10.2307/1467843

HENRY, CP., AMOROS, C. and BORNETTE, G. 1996. Species Traits and Recolonization Processes after Flood Disturbances in Riverine Macrophytes. Vegetatio, vol. 122, no. 1, p. 13-27. http://dx.doi. org/10.1007/BF00052812

JURAJDA, P., REICHARD, M. and SMITH, C. 2006. Immediate impact of an extensive summer flood of the adult fish assemblage on a channelized lowland river. Journal of Freshwater Ecology, vol. 21, no. 3, p. 493-501. http://dx.doi.org/10.1080/02705060. 2006.9665027
LANCASTER, J. 2000. Geometric scaling of microhabitat patches and their efficacy as refugia during disturbance. Journal of Animal Ecology, vol. 69, p. 442-457. http://dx.doi.org/10.1046/ j.1365-2656.2000.00407.x

LAKE, PS. 2000. Disturbance, patchiness, and diversity in streams. Journal of the North American Benthological Society, vol. 19, p. 573-592. http:// dx.doi.org/10.2307/1468118

LAKE, PS. 2013. Resistance, Resilience and Restoration. Ecological Management and Restoration, vol. 14, no. 1, p. 20-24. http://dx.doi.org/10.1111/emr.12016

LEE, HG. and BAE, YJ. 2011. Recovery of aquatic insect communities after a catastrophic flood in a Korean stream. Animal Cells and Systems, vol. 15, no. 2, p. 169-177. http://dx.doi.org/10.1080/1976 8354.2011.555121

LONGING, SD. and HAGGARD, BE. 2010. Biological assessment to support ecological recovery of a degraded headwater system. Environmental Management, vol. 46, p. 459-470. PMid:20703878. http://dx.doi.org/10.1007/s00267-010-9532-0

LONZARICH, DG., WARREN JR., ML. and LONZARICH, MRE. 1998. Effects of habitat isolation on the recovery of fish assemblages in experimentally defaunated stream pools in Arkansas. Canadian Journal Fisheries and Aquatic Sciences, vol. 55, p. 2141-2149. http://dx.doi.org/10.1139/ f98-113

LYTLE, DA. 2000. Biotic and abiotic effects of flash flooding in a montane desert stream. Archiv für Hydrobiologie, vol. 150, p. 85-100.

MADIGAN, MT., MARTINKO, JM., DUNLAP, PV. and CLARK, DP. 2009. Brock Biology of Microorganisms. Pearson San Francisco: Benjamin Cummings. $106 \mathrm{p}$.

MAGURRAN, AE. 2004. Measuring Biological Diversity. Oxford: Blackwell. 256 p.

MATTHAEI, CD., ARBUCKLE, CJ. and TOWNSEND, CR. 2000. Stable surface stones as refugia for invertebrates during disturbance in a New Zealand stream. Journal of the North American Benthological Society, vol. 19, p. 82-93. http://dx.doi. org/10.2307/1468283

MATTHEWS, WJ. 1998. Patterns in freshwater fish ecology. New York: Chapmann \& Hall. 756 p.

MALTCHIK, L. and PEDRO, F. 2001. Responses of aquatic macrophytes to disturbance by flash floods in a Brazilian semiarid intermittent stream. Biotropica, vol. 33, no. 4, p. 566-572.

MERRITT, RW. and CUMMINS, KW. 1996. An introduction to the aquatic insects of North America. Iowa: Kendall/Hunt Publ. Co. 862 p.

MOLLES JUNIOR, MC. 1985. Recovery of a stream invertebrate community from a flash flood in Tesuque creek, New Mexico. The Southwestern 
Naturalist, vol. 30, no. 2, p. 279-287. http://dx.doi. org/10.2307/3670741

MOTTA, RL. and UIEDA, VS. 2005. Food web structure in a tropical stream ecosystem. Austral Ecology, vol. 30, p. 58-73. http://dx.doi.org/10.1111/ j.1442-9993.2005.01424.x

MUNDAHL, ND. and HUNT, AM. 2011. Recovery of stream invertebrates after catastrophic flooding in southeastern Minnesota, USA. Journal of Freshwater Ecology, vol. 26, no. 4, p. 445-457.

NECCHI, O. 2004. Amostragem de macroalgas bentônicas. In BICUDO, CEM. and BICUDO, DC., eds. Amostragem em limnologia. São Carlos: RiMa. p. 167-175.

NIEMI, GJ., DEVORE, P., DETENBECK, N., TAYLOR, D., LIMA, A., PASTOR, J., YOUNT, JD. and NAIMAN, RJ. 1990. Overview of case studies on recovery of aquatic systems from disturbance. Environmental Management, vol. 14, no. 5, p. 571587. http://dx.doi.org/10.1007/BF02394710

NISLOW, KH., MAGILLIGAN, FJ., FOLT, CL. and KENNEDY, BP. 2002. Within-basin variation in the short-term effects of a major flood on stream fishes and invertebrates. Journal of Freshwater Ecology, vol. 17, no. 2, p. 305-318. http://dx.doi.org/10.108 0/02705060.2002.9663899

PASSOS, MIS., NESSIMIAN, JL. and JUNIOR, NF. 2007. Chaves para identificação dos gêneros de Elmidae (Coleoptera) ocorrentes no Estado do Rio de Janeiro, Brasil. Revista Brasileira de Entomologia, vol. 51, no. 1, p. 42-53.

PETERSON, JT. and BAYLEY, PB. 1993. Colonization rates of fishes in experimentally defaunated warmwater streams. Transactions of the American Fisheries Society, vol. 122, p. 199-207. http://dx.doi. org/10.1577/1548-8659(1993)122<0199:CROFI $\mathrm{E}>2.3 . \mathrm{CO} ; 2$

PICKETT, STA. and WHITE, PS. 1985. The ecology of natural disturbance and patch dynamics. New York: Academic Press. $472 \mathrm{p}$.

PIRES, AM., MAGALHÃES, MF., MOREIRA DA COSTA, L., ALVES, MJ. and COELHO, MM. 2008. Effects of an extreme flash flood on the native fish assemblages across a Mediterranean catchment. Fisheries Management and Ecology, vol. 15, p. 49-58. http://dx.doi.org/10.1111/j.13652400.2007.00570.x
POFF, NL., ALLAN, JD., BAIN, MB., KARR, JR., PRESTEGAARD, KL., RICHTER, BD., SPARKS, RE. and STROMBERG, JC. 1997. The Natural Flow Regime (a paradigm for river conservation and restoration). BioScience, vol. 47, no. 11, p. 769-784.

RESH, VH., BROWN, AV., COVICH, AP., GURTZ, ME., LI, HW., MINSHALL, GW., REICE, SR., SHELDON, AL., WALLACE, JB. and WISSMAR, RC. 1988. The role of disturbance in stream ecology. Journal of the North American Benthological Society, vol. 7, p. 433-455. http:// dx.doi.org/10.2307/1467300

SOUZA, LOI., COSTA, JM. and OLDRINI, BB. 2007. Odonata. In FROEHLICH, CG., org. Guia on-line: Identificação de larvas de insetos aquáticos do Estado de São Paulo. Available from: <http://sites.ffclrp.usp. br/aguadoce/guiaonline>.

STANLEY, PH., POWERS, SM. and LOTTIG, NR. 2010. The evolving legacy of disturbance in stream ecology: concepts, contributions, and coming challenges. Journal of the North American Benthological Society, vol. 29, no. 9, p.67-83. http:// dx.doi.org/10.1899/08-027.1

THOMSON, JR., LAKE, PR. and JONES, BJ. 2002. The effect of hydrological disturbance on the impact of a benthic invertebrate predator. Ecology, vol. 83, no. 3, p. 628-642. http://dx.doi.org/10.1890/00129658(2002)083[0628:TEOHDO]2.0.CO;2

TRIVINO-STRIXINO, S. and STRIXINO, G. 1994. Larvas de Chironomidae (Diptera) do Estado de São Paulo. Guia de identificação e diagnose de gêneros. São Carlos: PPG-ERN/UFSCAR.

WILLIAMS, DD. and HYNES, HBN. 1976. The Recolonization Mechanisms of Stream Benthos. Oikos, vol. 27, no. 2, p. 265-272. http://dx.doi. org/10.2307/3543905

WINEMILLER, KO., FLECKER, AS. and HOEINGHAUS, DJ. 2010. Patch dynamics and environmental heterogeneity in lotic ecosystems. Journal of the North American Benthological Society, vol. 29, no. 1, p. 84-99. http://dx.doi. org/10.1899/08-048.1

YOUNT, JD. and NIEMI, GJ. 1990. Recovery of lotic communities and ecosystems from disturbance - a narrative review of case studies. Environmental Management, vol. 14, no. 5, p. 547-569. http:// dx.doi.org/10.1007/BF02394709

Received: 10 July 2012 Accepted: 14 August 2013 